\title{
Theoretical study of the slow light in the assembly of helium atoms
}

\author{
A. G. Petrashen, N. V. Sytenko \\ ITMO University, Kronverkskiy, 49, Saint Petersburg, 197101, Russia \\ apetrashen@mail.ru,nsitenko@yandex.ru
}

PACS 32.8.QK,32.10.Dk, 32.80.-t

DOI 10.17586/2220-8054-2018-9-3-342-348

The problem of solving light was installed about thirty years ago and was widely considered in different approximations. In this paper, this process is investigated using both perturbation theory and numerical integration of the system of Liouville-Maxwell differential equations.

Keywords: coherent excitation, density matrix, Liouville equation.

Received: 14 March 2018

Revised: 29 March 2018

Final revision: 25 April 2018

\section{Introduction}

Effects caused by the quantum-mechanical coherence and interference of states have been extensively studied over the past few decades. Electromagnetically induced transparency give rise to a new direction of research, which deals with the fact that an anomalously strong dispersion of a weak beam in the field of a strong beam leads to a change in group velocity of weak beam photons. In the extreme case, this can lead to the stopping of low intensity photons, which in turn can lead to the creation of optical memory. The change in group velocity of a low intensity beam was discussed in the work of Harris et al. [1]. Later, this phenomenon was investigated in the papers of [2-4]. Among other works, in our opinion, special note should be made of the papers of Lukin $[5,6]$, where the author used the solution of the Heisenberg-Langevin equations for the density matrix in perturbation theory to analyze the dependence of the velocity on the parameters of the problem under consideration. In this paper, we will use both perturbation theory and numerical integration of differential equations to obtain expression for group velocity of radiation of a real atomic system. Similar methods of investigations were previously applied to the problem of influence of strong magnetic field on characteristics of emission of ensemble of helium atoms and the problem of possibility of lasering without inversion $[7,8]$. The results of such an approximate calculation will be checked against similar results obtained by numerical integration of the Liouville-Maxwell partial differential equations.

\section{The interaction of the atomic system with the field}

In this work, we will consider a system of three atomic states of the helium atom: $2^{3} P_{2}$ - (level $c$ ), $2^{3} P_{1}$ (level $a$ ) and $2^{3} S_{1}$ - (level $b$ ), and we will assume that levels " $a$ " and " $c$ " are coherently excited from level " $b$ " as according to the $V$-type excitation scheme. This trio of levels was chosen because the line that corresponds to a transition $2^{3} P-2^{3} S$ lies near the visible wavelength range, the upper ${ }^{3} P$ states relax relatively weakly, while the lower $2^{3} S_{1}$ state practically does not decay [9] and can be impact-excited from the ground state. The imposition of a time-dependent alternating electric field on the atomic system leads to charge displacement or, in other words, to the creation of currents, and, consequently, to the appearance an alternating electric and magnetic field. A joint influence of these quantities is described by a set of Maxwell equations, from which, using well-known transformations, it is not difficult to obtain equations that describes the mutual variation in the electric field vector $\mathbf{E}$ and polarization of the medium $\mathbf{P}$ :

$$
\nabla^{2} \mathbf{E}=\frac{\mu}{c^{2}} \frac{\partial^{2} \mathbf{E}}{\partial^{2} t}+\frac{4 \pi \mu}{c^{2}} \frac{\partial^{2} \mathbf{P}}{\partial^{2} t}
$$

From here forward, we will assume that the field and the polarization depend only on one spatial variable and time, and that the amplitudes of these magnitudes are slowly varying functions of their variables. Then, if we seek the amplitudes of these fields in the form of $E(x, t)=\mathcal{E}(x, t) e^{i(\omega t+k x)}+\mathcal{E}^{*}(x, t) e^{-i(\omega t+k x)}$ and $P(x, t)=\mathcal{P}(x, t) e^{i(\omega t+k x)}+\mathcal{P}^{*}(x, t) e^{-i(\omega t+k x)}$, then after substituting these functions into (1) and neglecting rapidly varying terms and higher-order derivatives of the field and the polarization vector amplitudes in the 
resulting expression, we obtain two equations which, if we introduce complex amplitudes for the field strength and polarization of the medium, can be reduced to one equation:

$$
\frac{\partial}{\partial \tau} \mathcal{E}(x, \tau)=-2 \pi \mu \omega i \mathcal{P}(x, \tau)
$$

It can be seen from the last relation that the imaginary part of the polarization vector characterizes field attenuation, i.e. absorption, while the real part characterizes dispersion. Note that in terms of its physical meaning, the magnitude of the polarization vector is proportional to the mean value of the dipole moment:

$$
\mathcal{P}_{a}=n_{0} \operatorname{Sp}\left(\rho_{a b} d\right)
$$

where $n_{0}$ is the particle's density and $\rho_{a b}(t)$ - is the correlation density matrix in the $a b$ arm.

It is well known, that the evolution of density matrix is described by the Liouville equation. In the case under consideration, this equation must be considered together with equation (2) because the external field enforced on the atomic system under consideration, leads to an induction of additional polarization fields. So one can say that evolution of our system describes by the the system of the Liouville-Maxwell equations.

To perform numerical calculations in this system of equations, it would be convenient to introduce dimensionless time $\tau=t / \tau_{0}$, where $\tau_{0}$ is the lifetime of the $2^{3} P$ state of the He atom, and the spatial variable $\kappa=x / c \tau_{0}$. In addition, as we continue, it would also be convenient to move from the quantities of the electric field strength to the dimensionless Rabi frequencies $\left(\Omega_{R}(\tau)\right)_{i b}=\mathcal{E}(\tau)_{i b} \tau_{0} e a_{0} / \hbar$ where the index $i$ numbers the corresponding arm of the $V$-type scheme. In these variables, the system of the Liouville-Maxwell equations will take the form:

$$
\begin{aligned}
\frac{d}{d \tau} \rho_{a a}(\tau, \kappa)= & -\gamma_{a a} \rho_{a a}(\tau, \kappa)-\frac{i\left(\Omega_{R}(\tau, \kappa)\right)_{a b}}{2}\left[\left(\frac{r_{q}}{a_{0}}\right)_{a b} \rho_{b a}(\tau, \kappa) e^{i \delta_{a} \tau}-\rho_{a b}(\tau, \kappa)\left(\frac{r_{q}}{a_{0}}\right)_{a b} e^{-i \delta_{a} \tau}\right] \\
\frac{d}{d \tau} \rho_{c c}(\tau, \kappa)= & -\gamma_{c c} \rho_{b b}(\tau, \kappa)-\frac{\left(\Omega_{R}(\tau, \kappa)\right)_{b c}}{2}\left[\left(\frac{r_{q}}{a_{0}}\right)_{c b} \rho_{b c}(\tau, \kappa) e^{i \delta_{c} \tau}-\rho_{c b}(\tau, \kappa)\left(\frac{r_{q}}{a_{0}}\right)_{b c} e^{-i \delta_{c} \tau}\right] \\
\frac{d}{d \tau} \rho_{b b}(\tau, \kappa)= & \gamma_{b b} \rho_{b b}(\tau, \kappa)-\frac{i\left(\Omega_{R}(\tau, \kappa)\right)_{a b}}{2}\left[\left(\frac{r_{q}}{a_{0}}\right)_{b a} \rho_{a b}(\tau, \kappa) e^{-i \delta_{c} \tau}-\rho_{b a}(\tau, \kappa)\left(\frac{r_{q}}{a_{0}}\right)_{a b} e^{i \delta_{c} \tau}\right] \\
& -\frac{i\left(\Omega_{R}(\tau, \kappa)\right)_{b c}}{2}\left[\left(\frac{r_{q}}{a_{0}}\right)_{b c} \rho_{c b}(\tau, \kappa) e^{-i \delta_{c} \tau}-\rho_{b c}(\tau, \kappa)\left(\frac{r_{q}}{a_{0}}\right)_{c b} e^{i \delta_{c} \tau}\right] \\
& +\frac{i\left(\Omega_{R}(\tau, \kappa)\right)_{b c}}{2} \rho_{a c}(\tau, \kappa)\left(\frac{r_{q}}{a_{0}}\right)_{c b} e^{-i \delta_{c} \tau}, \\
\frac{d}{d \tau} \rho_{a b}(\tau, \kappa)= & \gamma_{a b} \rho_{a b}(\tau, \kappa)-\frac{i\left(\Omega_{R}(\tau, \kappa)\right)_{a b}}{2}\left[\left(\frac{r_{q}}{a_{0}}\right)_{a b} \rho_{b b}(\tau, \kappa) e^{-i \delta_{a} \tau}-\rho_{a a}(\tau, \kappa)\left(\frac{r_{q}}{a_{0}}\right)_{a b} e^{i \delta_{a} \tau}\right] \\
& +\frac{i\left(\Omega_{R}(\tau, \kappa)\right)_{a b}}{2} \rho_{c a}(\tau, \kappa)\left(\frac{r_{q}}{a_{0}}\right)_{a b} e^{i \delta_{a} \tau}, \\
\frac{d}{d \tau} \rho_{c b}(\tau, \kappa)= & \gamma_{c b} \rho_{c b}(\tau, \kappa)-\frac{i\left(\Omega_{R}(\tau, \kappa)\right)_{b c}}{2}\left[\left(\frac{r_{q}}{a_{0}}\right)_{c b} \rho_{b b}(\tau, \kappa) e^{i \delta_{c} \tau}+\rho_{c c}(\tau, \kappa)\left(\frac{r_{q}}{a_{0}}\right)_{c b} e^{i \delta_{c} \tau}\right] \\
\frac{d}{d \tau} \rho_{a c}(\tau)= & -\gamma_{a c} \rho_{a c}(\tau)-\frac{i}{2}\left(\Omega_{R}(\tau, \kappa)\right)_{b c} \rho_{a b}(\tau, \kappa)\left(\frac{r_{q}}{a_{0}}\right)_{b c} e^{i \delta_{c} \tau}+\frac{i}{2}\left(\Omega_{R}(\tau, \kappa)\right)_{a b}\left(\frac{r_{q}}{a_{0}}\right)_{b c} \rho_{b c}(\tau, \kappa) e^{-i \delta_{a} \tau} \\
\frac{\partial}{\partial \tau}\left(\Omega_{R}\right)_{i}(\tau, \kappa)+ & \frac{\partial}{\partial \kappa}\left(\Omega_{R}\right)_{i}(\tau, \kappa)=-i \Omega_{M}^{2} \operatorname{Sp}\left(\rho_{b i}(\tau, \kappa)\right)\left(\frac{r_{b i}}{a_{o}}\right), \quad i=a_{c},
\end{aligned}
$$

where $\left(\frac{r_{q}}{a_{0}}\right)_{i, j}-$ is the matrix element of the $q$-th component of the operator $\hat{r} / a_{0}$, evaluated on the eigenfunctions of the $i$ and $j$ states (we assumed $q=0$ below for simplicity), $\Omega_{M}=\sqrt{4 \pi n_{0} \hbar \omega} \frac{\tau_{0} e a_{0}}{\hbar}\left\langle\frac{r}{a_{0}}\right\rangle-$ the the dimensionless value of the amplitude of the field oscillations that depends on mean value of the operator $\hat{r}$ in the $2^{3} P$ state. Substituting the values of the corresponding fundamental constants into the last relatione get $\Omega_{M}=0.001132 \sqrt{n_{0}}$, where, we recall, $n_{0}$ is the particle density expressed in inverse centimeters.

In the conclusion of this section, we note that in order to realize the process of coherent excitation in the $V$-type scheme, dipole moments must be first induced in the system. This can be done by a short seed pulse, which precedes the moment of the onset of excitation. 


\section{The variation of the phase velocity of a light wave during coherent excitation}

In this section, we will consider the problem of approximate solution of system (4) under the assumption that $\Omega_{b c} \gg \Omega_{b a}$ (later we shall omit second indexes at these values), a relatively slow change in the envelopes of field strengths and correlation density matrices, and also for time intervals not spaced very far from the origin of coordinates. We will proceed from the sixth equation of system (4). If we assume that the envelope of the correlation matrix $\rho_{a b}(\tau, \kappa)$ varies rather slowly, i. e. its derivative is zero, and the population of level $a$ is so small that it can be neglected, then it is possible to obtain a relation associating the population of level $b$ with the matrix that distributes the coherence between levels $a$ and $c$ :

$$
\rho_{a c}(\tau, \kappa)=\frac{e^{-i \tau\left(\delta_{a}-\delta_{c}\right)} \Omega_{a}(\tau, \kappa)}{\Omega_{c}(\tau, \kappa)}\left(\frac{r}{a_{0}}\right)_{a b} \rho_{b b}(\tau, \kappa) \Omega_{a}(\tau, \kappa)\left(\frac{r}{a_{0}}\right)_{c b}^{-1} .
$$

Hence, under the assumption that the Rabi frequency $\Omega_{a}$ is small in comparison with $\Omega_{c}$, we can obtain:

$$
\rho_{a b}(\tau, \kappa)=\left(-\frac{2 i e^{-i \delta_{c} \tau}}{\Omega_{c}(\tau, \kappa)}\right) \frac{\partial}{\partial \tau} \rho_{a c}(\tau, \kappa)\left(\frac{r}{a_{0}}\right)_{b c}^{-1} .
$$

From the last two relations it follows that:

$$
\rho_{a b}(\tau, \kappa)=\left(-\frac{2 i e^{-i \tau \delta_{c}}}{\Omega_{c}(\tau, \kappa)}\right) \frac{\partial}{\partial t}\left(\frac{e^{i \tau\left(\delta_{a}-\delta_{c}\right)} \Omega_{a}(\tau, \kappa)}{\Omega_{c}(\tau, \kappa)}\left(\frac{r}{a_{0}}\right)_{a b} \rho_{b b}(\tau, \kappa)\left(\frac{r}{a_{0}}\right)_{c b}^{-1}\right)\left(\frac{r}{a_{0}}\right)_{b c}^{-1} .
$$

Then, assuming $\delta_{a}=\delta_{c}$ after rather cumbersome, collations that we perform with program "Mathematica", one has the relation:

$$
\begin{gathered}
\frac{\partial}{\partial \tau}\left(\Omega_{R}\right)_{a}(\tau, \kappa)+\frac{\partial}{\partial \kappa}\left(\Omega_{R}\right)_{a}(\tau, \kappa)= \\
-i \Omega_{M}^{2} \operatorname{Sp}\left\{\left[\left(-\frac{2 i e^{-i \tau \delta_{c}}}{\Omega_{c}(\tau, \kappa)}\right) \frac{\partial}{\partial \tau}\left(\frac{\Omega_{a}(\tau, \kappa)}{\Omega_{c}(\tau, \kappa)}\left(\frac{r}{a_{0}}\right)_{a b} \rho_{b b}(\tau, \kappa)\left(\frac{r}{a_{0}}\right)_{c b}^{-1}\right)\left(\frac{r}{a_{0}}\right)_{b c}^{-1}\right]\left(\frac{r}{a_{0}}\right)_{b a}\right\} .
\end{gathered}
$$

If the Rabi frequency in the arm $b c$ is large and slowly varying, the last relation can be transformed to the form:

$$
\frac{1}{v} \frac{\partial}{\partial t} \Omega_{a}(\kappa, \tau)+\frac{\partial}{\partial x} \Omega_{a}(\kappa, \tau)=0
$$

Where, for the inverse phase velocity, one can write:

$$
\frac{1}{v}=\frac{1}{c}\left[1+2 \Omega_{M}^{2} \frac{\Omega_{a}(\tau, \kappa)}{\Omega_{c}^{2}(\tau, \kappa)} \operatorname{Sp}\left[\left(\frac{r}{a_{0}}\right)_{a b} \rho_{b b}(\tau, \kappa)\left(\frac{r}{a_{0}}\right)_{c b}^{-1}\left(\frac{r}{a_{0}}\right)_{b c}^{-1}\left(\frac{r}{a_{0}}\right)_{b a}\right]\right] .
$$

According to the properties of the matrix trace, from the last relation, one can obtain the expression equitable at the first order over the ratio $\Omega_{a} /\left(\Omega_{c}\right)^{2}$ :

$$
\frac{c}{v}-1=2 \Omega_{M}^{2} \frac{\Omega_{a}}{\Omega_{c}^{2}} \operatorname{Sp}\left(\left\langle\left(\frac{r}{a_{0}}\right)^{2}\right\rangle_{a} \rho_{b b}(\tau, \kappa)\left\langle\left(\frac{r}{a_{0}}\right)^{2}\right\rangle_{c}\right)
$$

where $\left\langle\left(\frac{r}{a_{0}}\right)^{2}\right\rangle_{i}(i=a, c)$ denotes the mean value of square of $r / a_{0}$ value, calculated over the wave functions of states $a$ and $c$. According to the fact that all values except $\Omega_{a}$ that are contained under the trace symbol are not negative, one can affirm that the relation between $c$ and $v$ depends on the sign of $\Omega_{a}$ : if $\Omega_{a}>0$ then $c>v$ and vice versa if $\Omega_{a}<0$.

On the Fig. 1 (curve 1) where for the ensemble of the helium atoms in $2^{3} P_{j}, j=1,2$ state the dependence of the value $1-v(t) / c$ on the time is represented one can see that the cure monotonically decreases over time.

In conclusion of this section, we wish to point out two things: firstly, the change in the velocity of a light wave due to the polarization of the medium during coherent excitation in the framework of the perturbation theory methods has been extensively studied and described in a large number of works (see, for example, [5]). The present study differs only in that it has been performed in a matrix form, which allows us to apply the formulas obtained herein to fine and hyperfine multiplets. Secondly, we obtained formula (11) by making rather serious assumptions, the main one of which, it seems to us, is the independence of the Rabi frequencies from time. 


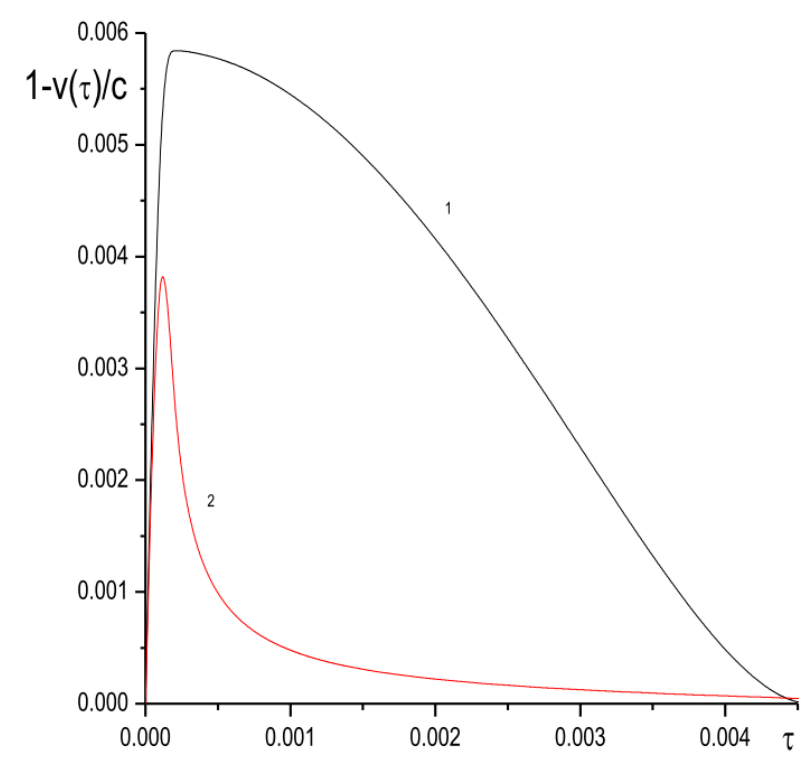

FIG. 1. Curves 1 and 2 illustrates the dependance of ratio $v / c$ obtained with perturbation theory and numerical solution of the Liouville-Maxwell equation

\section{Results of numerical calculations}

Before presenting the results of numerical calculations, we recall that each of the electric fields acting in the arms $a b$ and $c b$ is made up of two fields - the excitation field and the polarization field that is created in the process of excitation. Below we will assume that the excitation field acting in the arm $c b$ ("coupling field") is strong, whereas the "probe field" acting in the arm $a b$ is weak (one field which we examined was 100 times stronger than the other). When performing numerical calculations, we chose the values of relaxation constants according to [9].

Let us turn now to the last equation of system (4) describing the transition $b a$. It is known from the theory of differential equations [11] that such partial differential equation is equivalent to a system of ordinary differential equations of the type

$$
\frac{d x}{c \tau_{0}}=\frac{d \Omega}{-i \Omega_{M}^{2} \operatorname{Sp}\left(\rho_{a b} \frac{r_{a b}}{a_{0}}\right)}=d \tau .
$$

If we choose the dimensionless time $\tau$ as an independent variable, a general solution of last equation, can be written in the form:

$$
\begin{gathered}
x=\left(c \tau_{0}\right) \tau+C_{1}, \\
\Omega_{a}=-i \Omega_{M}^{2} \int \operatorname{Sp}\left(\rho_{a b} \frac{r_{a b}}{a_{0}}\right) d \tau+C_{2} .
\end{gathered}
$$

In order to find the magnitude of $\Omega_{a}$ - the polarization frequency in the arm $a b$, we need to solve system (4), calculate the value of the correlation density matrix $\rho_{a b}(\tau)$ for each value of $\tau$ and, in parallel, use Simpsons formula to calculate the integral (13). The results of the calculations are presented in the $3 D$-figure (Fig. 2). From this plot one can see that the Rabi polarization frequency has the form of a rather short pulse. Similar results were obtained earlier in $[3,4]$.

Note that when plotting Fig. 2, we assumed that spatial coordinates are linearly time-dependent, which, generally speaking, is not true even in the case under consideration, when system (4) is clearly independent of spatial coordinates. Nonlinear time dependence of spatial coordinates is explained by the fact that time dependence of these quantities is due to the correlation matrix $\rho_{a b}$, which is one of the solutions of system (4). In order to illustrate the effect of polarization of the medium on spatial coordinates, we numerically solved system (4), as a result of which the first two partial time derivatives of the Rabi frequency were determined. Further, if we write down formula (12) as:

$$
\frac{v(\tau)}{c}=\frac{\frac{d \Omega(\tau)}{d \tau}}{-i \Omega_{M}^{2} \operatorname{Sp}\left(\rho_{a b} \frac{r_{b a}}{a_{0}}\right)},
$$




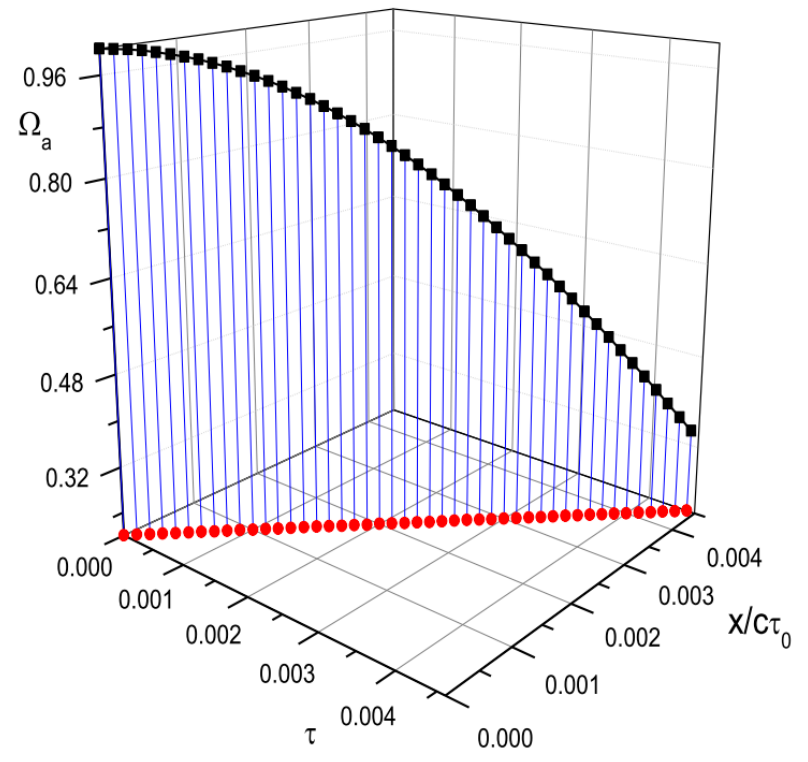

FIG. 2. Rabi frequency, in the arm $t a b$, as a function of spatial coordinates and time

and replace the total derivative in the numerator of the last expression with its truncated Taylor series, we obtain the expression:

$$
1-\frac{v}{c}=1-\frac{\frac{\partial \Omega_{a}}{\partial \tau}+\frac{1}{2} \frac{\partial^{2} \Omega_{a}}{\partial \tau^{2}} d \tau}{-i \Omega_{M}^{2} \operatorname{Sp}\left(\rho_{a b} d\right)}=\frac{\frac{1}{2} \frac{\partial^{2} \Omega_{a}}{\partial \tau^{2}} d \tau}{i \Omega_{M}^{2} \operatorname{Sp}\left(\rho_{b a} d_{a b}\right)}=-\frac{\operatorname{Sp}\left(\frac{d}{d t} \rho_{a b} d_{b a}\right) d \tau}{2 \operatorname{Sp}\left(\rho_{b a} d_{a b}\right)}
$$

By using the numerical integration of the system, we calculated the right-hand side of expression (15) as a function of time. In the process, it turned out that function $1-v(\tau) / c$ has a sharp maximum near the origin of coordinates and then decreases rapidly. The situation described is illustrated in Fig. 1 (curve 2), where, we recall, the first curve corresponds to the dependence of the relation under consideration determined by the approximate formula (11).

One of the most exciting challenges associated with the problem under consideration is the possibility of controlling the process of changing the magnitude of the group velocity of light. This problem in the framework of the approximate solution of the Liouville-Maxwell equation in theory is considered in detail in [4]. Below, we investigate this question in the framework of the approximations used in this paper.

We recall that, as noted earlier, that the sign of $\Omega a$ determines the ratio of the group velocity to the speed of light in vacuum. From general considerations, it is clear that the possibility of manipulating the magnitude of the speed of light should depend on individual properties of emitting particles. In the present paper, the individual properties of emitting particles included both in the parameters of the energy distribution of levels, and in the magnitudes of the dipole momentums, which, in turn, determine the Rabi frequency. The elements of the dipole moment matrix depend both on the polarization direction of the exciting radiation and on the wave functions of the initial and final states. We accurately calculated the angular parts of the matrix elements of the dipole moment matrix in both arms of the $V$-type scheme, after which, in view of the fact that radial wave functions are not generally known, each element of the dipole moment matrix in the arm $a b$ increased by $(\lambda=1,1.5,2)$ times.

We can see from Fig. 3, which presents the time dependence of the Rabi frequency generated in the arm $a b$ for different values of $\lambda$, that this magnitude depends quite heavily on the magnitude of the dipole moment. We further note that manipulating the velocity magnitude by changing the direction of the dipole moment induced in the arms of the $V$-type scheme is unlikely to give the expected effect. Such a change is expressed by similarity transformation and, as follows from formula (11), owing to the invariance of the matrix trace with respect to such transformation, the ratio of the phase velocity of light to the similar magnitude in vacuum will not change.

In order to manipulate the ratio $v / c$, from our point of view, it is convenient to use a weak magnetic field whose direction does not coincide with the direction of polarization of the radiation acting in the arms of the $V$-type scheme. (It will be recalled that the excitation direction fixes the axis $O Z$ of the laboratory coordinate system). Let us associate the axis $O X$ of this system with the direction of the weak magnetic field. Then the 


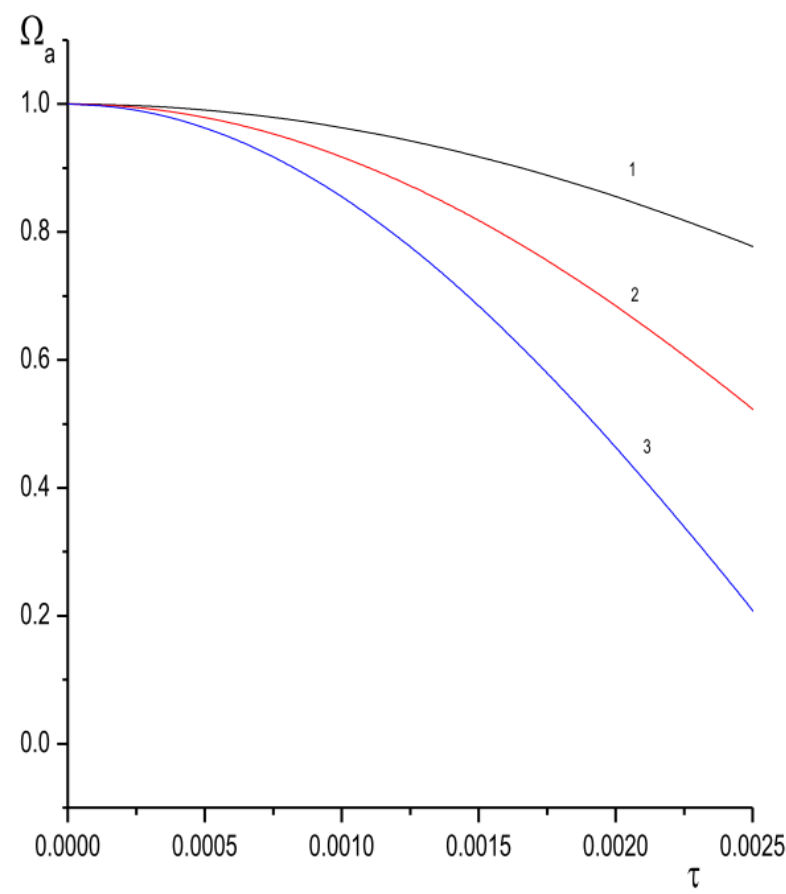

FIG. 3. Time dependence of the Rabi frequency generated in the arm $a b$ on the values of the dipole moment. Curves $1-3$ correspond to the values of $\lambda=1,1.5,2$

precession of the angular moment with respect to this direction will lead to a redistribution of the population of levels within each multiplet.

For mathematical description of the influence of a weak magnetic field along the $O X$ axis of the laboratory frame of reference on the population of fine sublevels of the $V$-type scheme under consideration, a commutator $\Omega_{L}\left[\rho, j_{x}\right]_{i, j}$ should be introduced into each line of the system of the Liouville-Maxwell differential equations (4) describing a change to the density matrix $\left.\left.\rho_{(} i, j\right),(i, j=a, b, c)\right)$, where $\Omega_{L}=\mu_{0} \mathcal{H} \tau_{0} / \hbar$ is the dimensionless Larmor frequency and then, by solving the aforementioned system, we can use formula (13) to determine the values of the correlation density matrices $\rho_{a b}(\tau)$ and $\rho_{c b}(\tau)$. Knowing the time dependence of these matrices, we can use formula (13) to determine the similar dependence for the polarization frequency. The above is illustrated in Fig. 4 that shows the dependence of this polarization frequency generated in the arm $c b$. It can be seen from the figure that as the magnitude of the magnetic field increases, the frequency of the generated polarization field increases, whereas the magnitude of this field decreases, which agrees with the model of the angular moment precessing in the magnetic field.

\section{Conclusion}

In this paper, we have considered the process of light propagation in a dispersive medium. In addition to the perturbation theory method, which was widely used earlier, in this work we performed numerical integration of the Liouville-Maxwell equations. All calculations, including analytical estimates, were performed in a matrix form, which allows for the application of the results of this paper to various fine and hyperfine multiplets. 


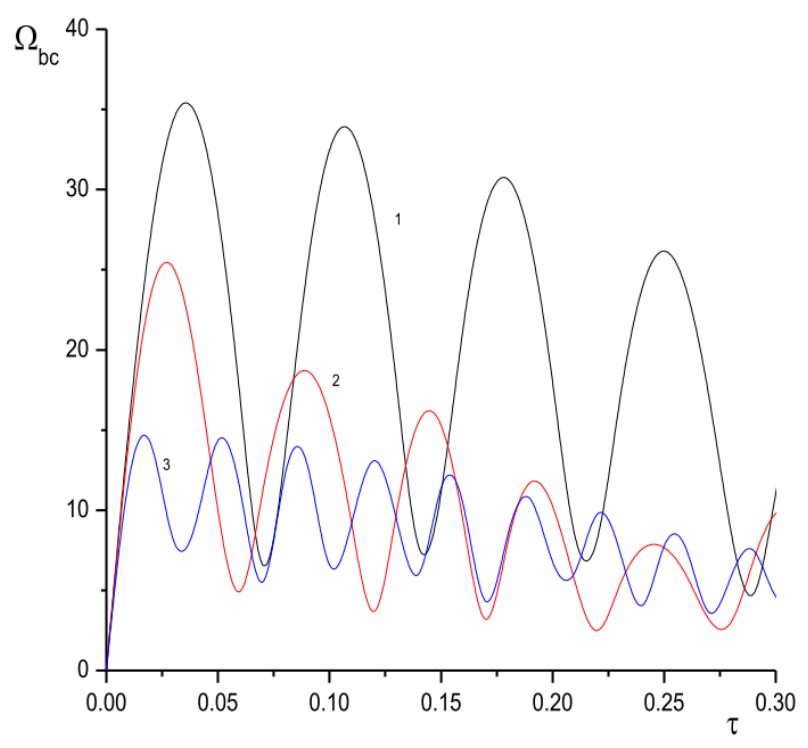

FIG. 4. Time dependence of the polarization frequency generated in the arm $c b$ for the values of the external magnetic field $\mathcal{H}=0,50,100$ Gs

\section{References}

[1] Harris S.E., Field J.E., Kasapi A. Disperse properties of electromagneticaly induced transparency. Phys. Rev. A, 1992, 46 (1), P. 29-32.

[2] Imamoglu A., Schmidt H., Woods G. Deutsch M. Strongly interacting photons in a nonlinear cavity. Phys. Rev. Lett., 1997, 79, P. 14671469.

[3] Alexandrov E.B., Zapassky V.S. In the "chase" of the slow light. Phys. Usp., 2006, 176 (10), P. 1093-1102.

[4] Zelinsky I.V., Mironov V.A. The electromagneticaly induced transperancy. JEPT, 2002, 121 (5), P. 1068-1079.

[5] Fleischhuer M., Lukin M.D. Dark-stated polarization in elecromagneticaly induced transperancy. Phys. Rev. Lett., 2000, 84 (22), P. 50945097.

[6] Lukin M.D. Trapping and manipulating photon states in atomic ensembles. Rev. Mod. Phys., 2003, 75, P. 457-472.

[7] Petrashen A.G., Sytenko N.V. Polarization characteristics of radiation of atomic ensemble under coherent excitation in the presence of the strong magnetic field. Nanosystems: Physics, Chemistry, Mathematics, 2015, 6 (1), P. 122-132.

[8] Petrashen A.G., Sytenko N.V. Laser generation without inversion on the levels of the Helium atoms. Nanosystems: Physics, Chemistry, Mathematics, 2017, 7 (2), P. 384-393.

[9] Frish S.E. Optical spectra of atoms. Moscow, 1963.

[10] NIST Atomic Spectra Database. URL: https://www.nist.gov/pml/atomic-spectra-database.

[11] Jackson J.D. Classical electrodynamics. Mir, Moscow, 1965, 702 p.

[12] Stepanov V.V. Course of differential equations. FIZMATLIT, Moscow, 1950. 